Western University

Scholarship@Western

2-1-2019

Multicentre, randomised clinical trial of paediatric concussion assessment of rest and exertion (PedCARE): a study to determine when to resume physical activities following concussion in children.

Andrée-Anne Ledoux

Nicholas J Barrowman

Kathy Boutis

Adrienne Davis

Sarah Reid

See next page for additional authors

Follow this and additional works at: https://ir.lib.uwo.ca/paedpub

Part of the Pediatrics Commons 


\section{Authors}

Andrée-Anne Ledoux, Nicholas J Barrowman, Kathy Boutis, Adrienne Davis, Sarah Reid, Gurinder Sangha, Ken J Farion, Kevin Belanger, Mark S Tremblay, Keith Owen Yeates, Carol DeMatteo, Nick Reed, and Roger Zemek 


\title{
Multicentre, randomised clinical trial of paediatric concussion assessment of rest and exertion (PedCARE): a study to determine when to resume physical activities following concussion in children
}

\author{
Andrée-Anne Ledoux, ${ }^{1}$ Nicholas J Barrowman, ${ }^{1}$ Kathy Boutis, ${ }^{2}$ Adrienne Davis, ${ }^{2}$ \\ Sarah Reid ${ }_{1}^{1,3}$ Gurinder Sangha, ${ }^{4}$ Ken J Farion, ${ }^{1,3}$ Kevin Belanger, ${ }^{1}$ Mark S Tremblay, \\ Keith Owen Yeates, ${ }^{5}$ Carol DeMatteo, ${ }^{6}$ Nick Reed, ${ }^{7}$ Roger Zemek, ${ }^{1,3}$ Pediatric \\ Emergency Research Canada PedCARE team
}

\begin{abstract}
'Children's Hospital of Eastern Ontario Research Institute, Ottawa, Canada ${ }^{2}$ Department of Pediatrics, Hospital for Sick Children, Toronto, Canada ${ }^{3}$ Department of Pediatrics, Children's Hospital of Eastern Ontario, University of Ottawa, Ottawa, Canada

${ }^{4}$ Pediatric Emergency Medicine, Children's Hospital London Health Sciences Centre, London, Ontario, Canada ${ }^{5}$ Department of Psychology, Alberta Children's Hospita Research Institute, University of Calgary, Calgary, Canada ${ }^{6} S$ chool of Rehabilitation Science, McMaster University, Hamilton, Canada 'Holland Bloorview Kids Rehabilitation Hospital, Toronto, Canada
\end{abstract}

\section{Correspondence to} Dr Roger Zemek, Children's Hospital of Eastern Ontario Research Institute, 401 Smyth Road, Ottawa, ON K1H 8L1, Canada; rzemek@cheo.on.ca

Received 26 April 2017 Revised 11 May 2017 Accepted 17 May 2017 Published Online First 12 July 2017

\section{ABSTRACT}

Introduction Rest until symptom-free, followed by a progressive stepwise return to activities, is often prescribed in the management of paediatric concussions. Recent evidence suggests prolonged rest may hinder recovery, and early resumption of physical activity may be associated with more rapid recovery postconcussion. The primary objective is to determine whether the early reintroduction of non-contact physical activity beginning 72 hours postinjury reduces postconcussive symptoms at 2 weeks in children following an acute concussion as compared with a rest until asymptomatic protocol. Methods and analysis This study is a randomised clinical trial across three Canadian academic paediatric emergency departments. A total of 350 participants, aged 10-17.99 years, who present within 48 hours of an acute concussion, will be recruited and randomly assigned to either the study intervention protocol (resumption of physical activity 72 hours postconcussion even if experiencing symptoms) or physical rest until fully asymptomatic. Participants will document their daily physical and cognitive activities. Follow-up questionnaires will be completed at 1, 2 and 4 weeks postinjury. Compliance with the intervention will be measured using an accelerometer (24 hours/day for 14 days). Symptoms will be measured using the validated Health and Behaviour Inventory. A linear multivariable model, adjusting for site and prognostically important covariates, will be tested to determine differences between groups. The proposed protocol adheres to the RCT-CONSORT guidelines.

Discussion This trial will determine if early resumption of non-contact physical activity following concussion reduces the burden of concussion and will provide healthcare professionals with the evidence by which to recommend the best timing of reintroducing physical activities.

Trial registration number Trial identifier ( Clinicaltrials.gov) NCT02893969.

\section{INTRODUCTION}

\section{Background information and scientific rationale}

To cite: Ledoux A-A Barrowman NJ, Boutis K, et al. Br I Sports Med 2019:53:195.
Concussion is defined as a complex pathophysiological process affecting the brain, induced by traumatic biomechanical forces. ${ }^{1}$ Paediatric concussion is an important and rapidly increasing health problem in the paediatric population, ${ }^{2-5}$ accounting for more than $95 \%$ of all traumatic brain injury (TBI) cases. ${ }^{6}$ Compared with adults, children and adolescents are at higher risk of concussion and take longer to recover. ${ }^{27}$ In fact, $30 \%$ of the paediatric population will suffer from persistent postconcussion symptoms at 1 month postinjury. ${ }^{8}$ Persistent postconcussive symptoms (PPCS) is a potentially disabling condition defined as persistence of somatic (eg, headache, dizziness and fatigue), cognitive (eg, forgetfulness and inattention) and other physical, psychological and behavioural changes lasting beyond 1 month $^{9}$ and persisting for months or years. ${ }^{2} 7$ 10-16 Persistence of these symptoms negatively affects the quality of life of both patients and their families. ${ }^{511} 17$ Having PPCS may reduce school attendance, academic performance and social encounters with peers due to removal from sports and recreational activities. ${ }^{11} 18-21$

With the hope of reducing the risk of PPCS, a widely used practice in paediatric concussion management is to prescribe cognitive and physical rest. $^{722-32}$ However, recently updated guidelines $(2017)^{133}$ recommend 24-48 hours of rest period before reintroducing gradually cognitive and physical activities in the child's routine. ${ }^{133} 34$ Despite these new recommendations, little evidence beyond expert opinion exists to guide healthcare professionals on how and when to best reintroduce physical activity to promote recovery and reduce the burden of PPCS for children and families. ${ }^{22} 3135-38$ The lack of evidence-based studies on rest and exertion may explain the wide practice variation by professionals in the management of paediatric concussion. $22363739-42$

Although evidence confirms that rest is important, ${ }^{43}$ recent studies have begun to suggest that protracted rest (ie, rest until full resolution of symptoms) may in fact negatively affect concussion recovery ${ }^{44} 45$ and lead to secondary symptoms of fatigue, depression and physiological deconditioning. ${ }^{46}$ A recent randomised controlled trial (RCT) demonstrated that strict cognitive rest for 5 days postinjury predisposed to worsening symptoms and delayed recovery as compared with 1-2 days cognitive rest followed by gradual return to normal cognitive activity. ${ }^{44}$ For physical rest, no 
comparative studies have been performed. Based on animal studies, early physical rehabilitation may enhance function and accelerate recovery following brain injury, depending on the intensity, frequency and type of activities. ${ }^{45} 47$ Pilot evidence from symptomatic individuals supports the benefits from gradual resumption of aerobic exercise for symptom recovery, provided there is no increased risk of further head trauma. ${ }^{48-50} \mathrm{~A}$ recent cohort study suggests that children who resume physical activity at 1 week postinjury are at lower risk of PPCS compared with children who abstained from physical activity for longer periods. $^{51}$

Although early resumption of light physical activity, without concomitant risk of reinjury, may be beneficial following concussion, further research is needed to clinically confirm these results and to determine the best timing of initiation. ${ }^{33} 52$ The purpose of this randomised clinical trial is to investigate whether early reintroduction of physical activity can reduce PPCS in a paediatric population. Determining if earlier resumption of non-contact physical activity is associated with superior recovery may significantly affect the well-being of millions of children and families worldwide and cause a paradigm shift in concussion management, while at the same time mitigating the undesirable effects of deconditioning associated with rest until full resolution of symptom recommendations.

\section{Study objectives}

\section{Primary research objective}

The purpose of this study is to investigate whether early reintroduction of non-contact, physical activity at 72 hours postinjury reduces the degree of PPCS at 2 weeks in children aged 10 through 17.99 years following an acute concussion as compared with a rest until symptom resolution protocol, defined as stepwise resumption of activity only once fully asymptomatic, as per the Zurich consensus return-to-play protocol. ${ }^{7}$

\section{Secondary research objectives}

In this group of children, among those initiating physical activities at 72 hours versus rest until symptom resolution, we will examine the following:

1. Differences between groups in symptoms at 1 and 4 weeks follow-up

2. Differences between groups in health-related quality of life (including school and sports participation) and functional outcomes at 2 and 4 weeks

3. Differences between groups in adverse events (eg, total number)

4. Differences between groups in sleep variables (eg, duration)

5. Intensity, duration and type of physical activity associated with recovery (in all patients)

6. Long-term healthcare use (using linked provincial health administrative data)

7. Incidence and risk factors for long-term concussion-related comorbidities (using linked provincial health administrative data)

\section{Hypothesis}

Non-contact, aerobic physical activities resumed in a standardised, incremental fashion at 72 hours postinjury are hypothesised to result in superior recovery following paediatric concussion as compared with a rest until resolution protocol (graduated return-to-play initiated only after full symptom resolution).

\section{METHODS}

\section{Trial design}

The proposed study is a multicentre, equal randomisation, parallel-group study conducted in Canada (three sites). Two groups will be studied: (1) intervention (early physical activity) group: early gradual resumption of non-contact, aerobic physical activity initiated 72 hours postinjury even if children are experiencing symptoms; and (2) rest until symptom resolution group: graduated return to physical activity initiated only after full resolution of symptoms based on the Zurich return-to-play guidelines. ${ }^{7} \mathrm{~A}$ schematic diagram of the trial design, procedures and stages, can be viewed in figure 1; the proposed protocol adheres to the RCT-CONSORT research methods and the SPIRIT checklist.

\section{Study setting}

This study will include three Pediatric Emergency Research Canada (PERC) network hospitals: Children's Hospital of Eastern Ontario (Ottawa; coordinating centre), The Hospital for Sick Children (Toronto) and Children's Hospital London Health Sciences Centre (London). These centres constitute 3 of the 12 paediatric hospitals in Canada that are active PERC members and have a strong clinical research infrastructure with experience in recruiting patients for large clinical trials. ${ }^{53}$ Research ethics boards (REB) have provided written approval from all three sites.

\section{Eligibility criteria}

\section{Inclusion criteria}

Participants presenting to one of the study hospital emergency departments after sustaining a direct or indirect head injury will be eligible if they (1) are aged 10 through 17.99 years; (2) are diagnosed with a concussion, defined by Berlin consensus statement; (3) sustained the injury in the previous 48 hours; and (4) are proficient in English or French.

\section{Exclusion criteria}

Participants will be excluded if they present with traumatic head injuries with any of the following: emergency department (ED) Glasgow Coma Scale (GCS) score $\leq 13$; abnormality on standard neuroimaging studies, ${ }^{7}$ including positive head CT findings (neuroimaging is not required, but may be performed if clinically indicated); neurosurgical operative intervention, intubation or intensive care required; multisystem injuries with treatment requiring hospital admission, operating room or procedural sedation in ED (hospital admission for observation or management of ongoing concussion symptoms is not an exclusion criteria); severe, chronic neurological disorder or developmental delay resulting in communication difficulties; intoxication at the time of ED presentation as per clinician judgement; no clear history of trauma as primary event (eg, seizure, syncope or migraine); inability to resume physical activities (eg, fractured extremity or other concomitant injuries); inability to obtain a proper written informed consent/assent (eg, language barriers, absence of parental authority, developmental delay, intoxication, patient too confused to consent); or legal guardian not present (certain forms need to be completed by parents/legal guardians).

\section{Intervention protocols}

Experimental group description: intervention (early physical activity) group

The intervention group and the control group differ in two important ways: (1) timing of the reintroduction of physical activities and (2) accommodation of ongoing symptoms for 


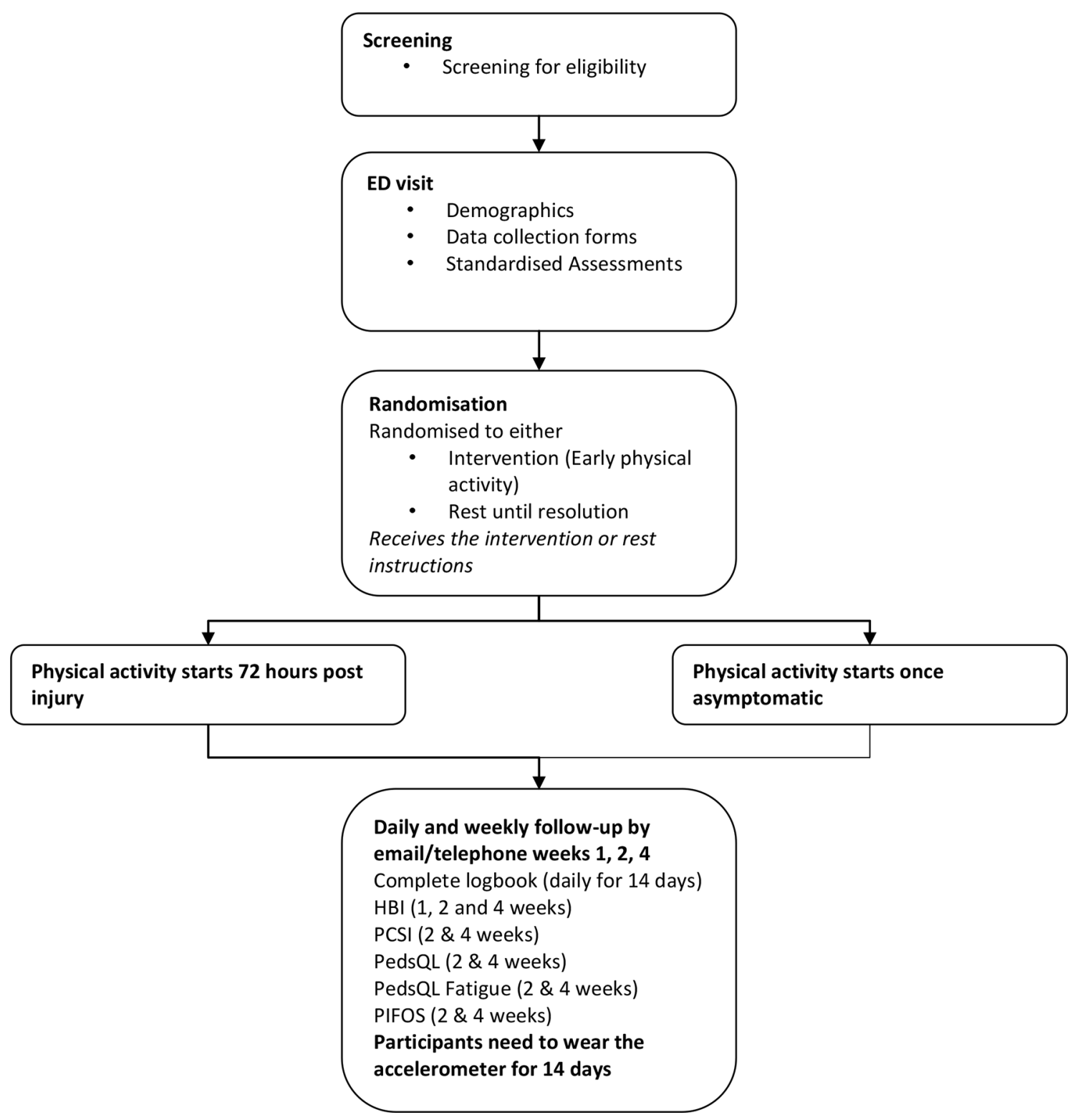

Figure 1 Study Procedure Flow Diagram. ED, emergency department ; HBI, Health and Behaviour Inventory; PCSI, Post-Concussion Symptom Inventory; PedsQL, Pediatric Quality of Life Inventory; PedsQL Fatige, PedsQL Multidimensional Fatigue Scale; PIFOS, Pediatric Injury Functional Outcome Scale.

both initiation and progression of return-to-play protocols. The experimental intervention (table 1 ) is based on the model for multimodal activity-exertion symptom management in children with concussion. ${ }^{55}$ After an initial period of full physical rest (stage 0 ), patients allocated to the intervention group will initiate light aerobic exercise (eg, 15 min walk; step 1) 72 hours postinjury (table 1 ). Physical activity will be introduced in a systematic and progressive manner, even if children are experiencing symptoms, starting at a low level of intensity such that it does not significantly increase symptoms and that the symptoms remain tolerable as per patient report.

A symptom-limited approach will be used to progressively increase activity duration and intensity as long as symptoms are well tolerated, with a minimum of 24 hours between each step. Patients will document the type of activity, its duration and intensity level [using the Pictorial Children's Effort Rating Table (PCERT)], ${ }^{56}$ along with preactivity/postactivity symptom severity. If symptoms become intolerable during or within $30 \mathrm{~min}$ following exercise (defined as much worse on the fifth and sixth questions of the logbook survey), children should return to the last well-tolerated level the next day and only attempt activity progression again after 24 hours. If children are unable to tolerate the initial step of the progression ( 15 min walk), they will be encouraged to attempt the walk again the next day.

\section{Control group description: physical activity following} symptom resolution (rest until symptom resolution) group Children allocated to the control group will be assigned to a rest until symptom resolution protocol (table 2). Based on the Zurich return-to-play guidelines, children will refrain from any physical activity until full resolution of all concussion symptoms (stage $0) .^{722-30}$ When asymptomatic, children may initiate walking (stage 1). If asymptomatic, activity intensity and duration will be increased in a stepwise fashion, with a minimum of 24 hours between each stage. If symptoms develop at any stage of the 
Table 1 Interventional (early physical activity) group protocol

\begin{tabular}{|c|c|c|c|c|}
\hline Stage & Description & Functional exercise & Objective & Criteria to move to next stage \\
\hline 0 & No physical activity & Symptom limited physical rest. & Recovery-72 hours postinjury & \\
\hline 1 & Walking & $\begin{array}{l}\text { Walking for } 15 \text { continuous minutes once a day at low } \\
\text { intensity [level three on the Pictorial Children's Effort } \\
\text { Rating Table (PCERT)]. No resistance training, weight } \\
\text { lifting or activities risking collision or falls. }\end{array}$ & $\begin{array}{l}\text { Initiate movement } \\
\text { Level of physical activity: very, very easy } \\
\text { to easy }\end{array}$ & $\begin{array}{l}\text { To move to stage } 2 \text { : } \\
\text { You have reached level } 3 \text { of the PCERT }\end{array}$ \\
\hline 2 & Light aerobic exercise & $\begin{array}{l}\text { Progression of activities using one of the following of } \\
\text { your choice per day: } \\
\text { - Type (eg, walking up and down the stairs, jogging/ } \\
\text { running, swimming, stationary cycling). } \\
\text { No resistance training, or activities risking collision or } \\
\text { falls. }\end{array}$ & $\begin{array}{l}\text { Increase heart rate } \\
\text { Level of physical activity: easy to just } \\
\text { feeling a strain }\end{array}$ & $\begin{array}{l}\text { To move to stage 3: } \\
\text { You have reached level } 4 \text { of the PCERT }\end{array}$ \\
\hline 3 & $\begin{array}{l}\text { Higher intensity/sport- } \\
\text { specific exercise }\end{array}$ & $\begin{array}{l}\text { Skating drills in ice hockey, running drills in soccer. } \\
\text { No activities risking collision or falls. }\end{array}$ & $\begin{array}{l}\text { Increase movement } \\
\text { Level of physical activity: starting to get } \\
\text { hard to getting quite hard }\end{array}$ & $\begin{array}{l}\text { To move to stage 4: } \\
\text { You have reached level } 6 \text { of the PCERT }\end{array}$ \\
\hline 4 & $\begin{array}{l}\text { High-intensity exercise/ } \\
\text { non-contact training } \\
\text { drills }\end{array}$ & $\begin{array}{l}\text { When sport-specific exercises are well tolerated, } \\
\text { progression to more complex training drills (eg, passing } \\
\text { drills in football and ice hockey). } \\
\text { Maximum level eight on the PCERT. } \\
\text { May start progressive resistance training. }\end{array}$ & $\begin{array}{l}\text { Increase exercise and improve } \\
\text { coordination } \\
\text { The level of physical activity is hard to } \\
\text { very hard }\end{array}$ & $\begin{array}{l}\text { To move to stage } 5 \text { : } \\
\text { You have reached level } 8 \text { of the PCERT AND } \\
\text { you have been cleared by a doctor }\end{array}$ \\
\hline \multicolumn{5}{|c|}{$\begin{array}{l}\text { Visit medical provider for clearance when asymptomatic at intensity level eight or higher, only progress to step five if medical clearance has been obtained and you are fully back } \\
\text { at school }\end{array}$} \\
\hline 5 & Full-contact practice & Participate in normal training activities & $\begin{array}{l}\text { Restore confidence and assess } \\
\text { functional skills by coaching staff }\end{array}$ & $\begin{array}{l}\text { To move to stage } 6 \text { : } \\
\text { Fully back to preinjury performance }\end{array}$ \\
\hline 6 & $\begin{array}{l}\text { Return to full } \\
\text { competition }\end{array}$ & Normal game play & & \\
\hline
\end{tabular}

Table 2 Rest until symptom resolution group protocol

\begin{tabular}{|c|c|c|c|c|}
\hline \multicolumn{2}{|c|}{ Rehabilitation stage } & \multirow{2}{*}{$\begin{array}{l}\text { Functional exercise } \\
\text { Symptom limited: physical rest }\end{array}$} & \multirow{2}{*}{$\begin{array}{l}\text { Objective } \\
\text { Recovery }\end{array}$} & \multirow{2}{*}{$\begin{array}{l}\text { Criteria to move to the next stage } \\
\text { To move to stage } 1: \\
\text { no more symptoms }\end{array}$} \\
\hline 0 & No physical activity & & & \\
\hline 1 & Walking & $\begin{array}{l}\text { Walking for } 15 \text { continuous minutes once a day } \\
\text { at low intensity [level three on the Pictorial } \\
\text { Children's Effort Rating Table (PCERT)]. No } \\
\text { resistance training, weight lifting or activities } \\
\text { risking collision or falls. }\end{array}$ & $\begin{array}{l}\text { Initiate movement } \\
\text { Level of physical activity: very, very easy to } \\
\text { easy }\end{array}$ & $\begin{array}{l}\text { To move to stage } 2 \text { : } \\
\text { No more symptoms } \\
\text { If you have symptoms, go back to stage } \\
0 . \text { Only progress when you have been } \\
\text { asymptomatic for } 24 \text { hours. }\end{array}$ \\
\hline 2 & Light aerobic exercise & $\begin{array}{l}\text { Walking, swimming or stationary cycling } \\
\text { keeping intensity to level } 1,2 \text { or } 3 \text { of the } \\
\text { PCERT. } \\
\text { No resistance training. }\end{array}$ & $\begin{array}{l}\text { Increase heart rate } \\
\text { Level of physical activity: easy to just feeling } \\
\text { a strain }\end{array}$ & $\begin{array}{l}\text { To move to stage } 3 \text { : } \\
\text { No symptoms } \\
\text { If you have symptoms, go back to stage } \\
\text { 1. Only progress when you have been } \\
\text { asymptomatic for } 24 \text { hours. }\end{array}$ \\
\hline 3 & $\begin{array}{l}\text { Higher intensity/sport- } \\
\text { specific exercise }\end{array}$ & $\begin{array}{l}\text { Skating drills in ice hockey, running drills in } \\
\text { soccer. } \\
\text { No activities risking collision or falls. }\end{array}$ & $\begin{array}{l}\text { Increase movement } \\
\text { Level of physical activity: starting to get hard } \\
\text { to getting quite hard }\end{array}$ & $\begin{array}{l}\text { To move to stage } 4 \text { : } \\
\text { No symptoms } \\
\text { If you have symptoms, go back to stage } \\
2 \text {. Only progress when you have been } \\
\text { asymptomatic for } 24 \text { hours. }\end{array}$ \\
\hline 4 & $\begin{array}{l}\text { High-intensity exercise/ } \\
\text { non-contact training } \\
\text { drills }\end{array}$ & $\begin{array}{l}\text { Progression to more complex training drills, } \\
\text { for example, passing drills in football and ice } \\
\text { hockey. } \\
\text { May start progressive resistance training. }\end{array}$ & $\begin{array}{l}\text { Increase exercise and improve coordination } \\
\text { The level of physical activity is hard to very } \\
\text { hard }\end{array}$ & $\begin{array}{l}\text { To move to stage } 5 \text { : } \\
\text { No symptoms and medical clearance is } \\
\text { needed } \\
\text { If you have symptoms, go back to stage } \\
\text { 3. Only progress when you have been } \\
\text { asymptomatic for } 24 \text { hours. }\end{array}$ \\
\hline \multicolumn{5}{|c|}{$\begin{array}{l}\text { Visit medical provider for clearance when asymptomatic at intensity level eight or higher, only progress to step five if medical clearance has been obtained and you are fully } \\
\text { back at school }\end{array}$} \\
\hline 5 & Full-contact practice & $\begin{array}{l}\text { Participate in normal training activities } \\
\text { following medical clearance }\end{array}$ & $\begin{array}{l}\text { Restore confidence and assess functional skills } \\
\text { by coaching staff }\end{array}$ & $\begin{array}{l}\text { To move to stage } 5 \text { : } \\
\text { No symptoms } \\
\text { If you have symptoms, go back to stage } \\
4 \text {. Only progress when you have been } \\
\text { asymptomatic for } 24 \text { hours. } \\
\text { Fully back to preinjury performance }\end{array}$ \\
\hline 6 & Return to play & Normal game play & & \\
\hline
\end{tabular}


protocol, children should drop back to the previous asymptomatic stage and try to progress again after having been asymptomatic for 24 hours.

\section{Common protocol description}

Children in both groups must refrain from any activities that increase the risk of reinjury (drills with body contact or that risk falls) until fully asymptomatic and cleared by their primary care or other medical provider. Finally, children will be counselled to follow the cognitive rest recommendations based on the Zurich Acute Concussion Evaluation (ACE) return-to-learn guidelines ${ }^{7}$ and recommendations provided by Iverson and Gioia. ${ }^{57}$ Briefly, this is a six-stage model where participants return gradually to school with proposed accommodations (ie, no return to school, at home; return to school with partial day (1-3 hours); full day with maximal support needed through the day; full day with moderate support; full day with minimal support; and full return with no supports needed).

\section{Participant compliance with the study intervention}

Compliance to the treatment will be measured objectively with an activity monitor device (Actical Accelerometer; Phillips Respironics, Bend, Oregon, USA). The device monitors movement (ie, frequency and intensity) in selected time-stamped epochs of $1 \mathrm{~min}$ and will be worn by all participants 24 hours a day for 14 days following enrolment. Final data points will be collected on day 14. Child and/or caregiver logbook report (telephone/ survey) will serve as the primary source of documentation.

\section{Outcomes}

Primary outcome and primary outcome measure

The primary aim of this randomised clinical trial is to evaluate whether early reintroduction of physical activity reduces the degree of postconcussive symptoms at 2 weeks. Symptoms will be measured using the Health and Behaviour Inventory (HBI). ${ }^{9}$ The HBI has been adopted as a core measure in the NIH Common Data Elements for Paediatric Traumatic Brain Injury. ${ }^{58}$ This measure is a 20 -item self-report questionnaire using a fourpoint Likert scale rating of symptom frequency, yielding separate scores for cognitive and somatic symptom scales and a total score ranging from 0 to 60 . The scale has good construct validity, reliability and was validated for children and adolescents $8-15$ years of age. ${ }^{9}$

Patients in the experimental group are hypothesised to have a 20\% lower total HBI score when compared with those in the control group at 2 weeks.

\section{Secondary outcomes and secondary outcome measures}

Several secondary outcomes are of interest:

- PPCS rates between the groups. The International Classification of Diseases, 10th Revision (ICD-10), defines PPCS as an increase from perceived preconcussion baseline of three or more concussion symptoms at 28 days following injury. ${ }^{59}$ For the purpose of this secondary outcome, PPCS case will be defined as an increase from preconcussion baseline of $\geq 3$ symptoms on both the HBI and the Postconcussion Symptom Inventory (PCSI), at 1, 2 and 4 weeks postinjury.

a. The Retrospective Health and Behaviour Inventory (rHBI) will be used to collect preinjury symptoms, and the HBI will be used to collect the postinjury symptom score. The rHBI is a 20 -item assessment answered by parents in the ED. These 20 items are exactly the same questions as within the HBI, except slightly worded differently. The assessment has good construct validity and good inter-rater reliability. ${ }^{9}$

b. For confirmatory purposes, we will use another instrument, the PCSI, ${ }^{60-62}$ to assess PPCS. The PCSI ${ }^{60} 63$ is a validated, comprehensive, self-administered instrument, and only one of two measures applicable to younger children with published validity and reliability data. ${ }^{60-62}$ For the purpose of this study, the PCSI preadolescent scale version (18-item, three-point scale), encompassing physical, cognitive, emotional and sleep domains will be used for all the children in the study. This specific version has demonstrated excellent internal consistency $(\mathrm{r}=0.87$ for children aged 8-12 years). The assessment will be included in the second and fourth week follow-ups.

- Quality of life. Delayed recovery from concussion has been shown to affect the quality of life of the patient and the family. ${ }^{1118}$ To assess whether early reintroduction of physical activity can prevent declines in health-related quality of life, the Paediatric Quality of Life Inventory version 4.0 (PedsQL) $^{64}{ }^{65}$ and PedsQL Multidimensional Fatigue Scale, will be used. Both assessments are reliable and valid measures of health-related quality of life in healthy children and adolescents and those with acute and/or chronic health conditions. ${ }^{65-68}$ For this study, the child's version (ages 8-12 and 13-18 years) will be used and the two measures will be administered during second and fourth week of follow-up.

a. The PedsQL is a 23-item, five-point scale, covering four domains: physical, emotional, social and school and takes approximately $4 \mathrm{~min}$ to complete.

b. The PedsQL Multidimensional Fatigue Scale is an 18 -item, five-point scale designed to measure fatigue in paediatric patients and comprises the General Fatigue Scale (six items), Sleep-Rest Fatigue Scale (six items) and Cognitive Fatigue Scale (six items).

Functional outcome. The long-term effects of a concussion have been shown to affect both psychological and physical outcomes. Communication and self-care skills seem to be vulnerable to disruption after a concussion. ${ }^{69}$ Academic performance is also hampered, with high rates of failure and need for academic support previously reported. ${ }^{70-73}$ Thus, studying functional outcomes (motor, cognitive, communication skills, social-emotional, self-care and physical) postconcussion is important.

a. The Pediatric Injury Functional Outcome Scale (PIFOS $)^{72}$ will be used to evaluate functional outcomes and determine if early physical activity can reduce the occurrence of poor functional outcomes. The PIFOS is a 26-item structured interview and is completed by caregivers. The assessment as good internal consistency $(\alpha=0.90$ $.93)$ and inter-rater reliability $(\alpha=0.90)$ for parents with children 3-15 years of age. ${ }^{72}$ It elicits ratings of motor skills, daily living skills, communication skills, cognition, social-emotional functioning, physical changes and academic functioning.

- Physical activity. Exploratory research into the ideal duration, intensity and type of physical activity that reduces the risk of PPCS. Physical activity will be recorded using a logbook and objectively measured via accelerometer data.

a. The patient will complete a daily logbook, the primary measure for assessing physical activity. This is a sevenitem survey and questions relate to the child's concussion symptoms, daily physical activities (including type of activity, duration and intensity using the validated PCERT $)^{56}$, return to school progression and severity of 
symptoms during and postactivity.

b. Actical accelerometer model z (Philips Respironics, Bend, Oregon, USA) will be used. The Actical provides an objective measurement of movement and sedentary time demonstrated by the participant. The device is capable of measuring or calculating raw acceleration, activity counts, energy expenditure and physical activity intensity. The device can store up to $32 \mathrm{MB}$ of data (ie, 194 days on the Epoch mode of $1 \mathrm{~min})$. The battery provides power for 180 days. The Actical accelerometer provides valid and reliable measures of movement behaviours. ${ }^{7475}$ Data collection and preprocessing (ie, cleaning and reduction) will follow established procedures for wear time criteria. ${ }^{7677}$

c. Safety. We will examine the safety of early resumption of physical activity. Three extra questions will be added to the daily activity logbook survey (longer version), for the 1, 2 and 4 week follow-up. These safety questions address participants current versus preinjury activity levels, whether they have played contact sports since their injury, and if they have had any adverse events (ie, unscheduled visit to the ED or your primary provider due to exacerbation of symptoms).

- Long-term outcome. Concussion affects the overall health of individuals for years to come. To better understand the health-related and economic effect of paediatric concussions, the long-term healthcare use of study participants will be investigated in a future substudy. Using the Institute for Clinical Evaluative Sciences (ICES) Linkage services and Ontario Health Insurance Plan (OHIP) number, long-term healthcare use (ie, health information from healthcare services that the patient has used over the years) will be collected. These variables will help determine risks for concussion-related comorbidities, the effect of concussions on school performances, and help establish the economic effect of paediatric concussions. All legal residents of the province of Ontario with an Ontario health card are contained and tracked within health administrative data. These health administrative databases are maintained by ICES through a comprehensive data sharing agreement with the Ontario Ministry of Health and Long-Term Care. ICES take several steps to ensure that all collected personal health information remains secure, confidential and safeguarded. Personal health information can be collected from multiple sources under this clause: health information custodians including doctors, hospitals, longterm care facilities, community care access centres, pharmacies, laboratories/specimen collection centres, ambulance services, special care services and community health/community mental health programs, among others.

Please refer to table 3 for the specific time points of each outcome measure.

\section{ED visit measurements}

\section{Case report forms}

Information about children's injuries will be collected from medical records and medical personnel using a standardised case report form. Details regarding the injury and acute signs and symptoms of concussion (ie, loss of consciousness; GCS scores; mechanism of injury; neurological status, and other clinical features) will be collected by research assistants (RA), with clarification by physicians as necessary. Data will be verified by the site investigator.

Demographic data (such as household annual income and education level) will be used as a measure for socioeconomic status (SES). SES will be collected to assess whether randomisation is equally distributed in both groups. If SES is not equally represented in the sample, it will be controlled for in our

Table 3 Schedule of enrolment, interventions and assessments.

\begin{tabular}{|c|c|c|c|c|c|c|c|}
\hline \multirow{3}{*}{$\begin{array}{l}\text { ED } \\
\text { Time point }\end{array}$} & \multirow[b]{3}{*}{ Enrolment } & \multirow[b]{3}{*}{ Allocation } & \multicolumn{5}{|c|}{ Study period } \\
\hline & & & \multicolumn{5}{|c|}{ Postallocation } \\
\hline & & & Day ${ }_{1-6}$ & Day $_{7}$ & Day $_{8-13}$ & Day $_{14}$ & Day $_{28}$ \\
\hline \multicolumn{8}{|l|}{ Enrolment: } \\
\hline Eligibility screen & $\mathrm{X}$ & & & & & & \\
\hline Informed consent & $\mathrm{X}$ & & & & & & \\
\hline Allocation & & $\mathrm{X}$ & & & & & \\
\hline \multicolumn{8}{|l|}{ Interventions: } \\
\hline Physical activity & & & & & & & \\
\hline Rest until symptom resolution & & & & & & & \\
\hline \multicolumn{8}{|l|}{ Assessments: } \\
\hline Case report (acute signs and symptoms) & & $x$ & & & & & \\
\hline Physical activity & & $\mathrm{X}$ & & & & & \\
\hline BESS & & $X$ & & & & & \\
\hline $\mathrm{rHBI}$ & & $\mathrm{X}$ & & & & & \\
\hline $\mathrm{HBI}$ & & $\mathrm{x}$ & & $\mathrm{x}$ & & $x$ & $x$ \\
\hline PCSI & & & & & & $X$ & $X$ \\
\hline PedsQL & & & & & & $\mathrm{x}$ & $x$ \\
\hline PedsQL Fatigue Scale & & & & & & $X$ & $X$ \\
\hline PIFOS & & & & & & $\mathrm{X}$ & $x$ \\
\hline Activity logbook & & & $\mathrm{X}$ & $\mathrm{X}$ & $x$ & $\mathrm{X}$ & $x$ \\
\hline Accelerometer (Actical) & & & $X$ & $X$ & $X$ & $X$ & \\
\hline
\end{tabular}

BESS, balance error scoring system; HBI, Health and Behaviour Inventory; PCSI, Postconcussion Symptom Inventory; PedsQL, Paediatric Quality of Life Inventory; PedsQL Multidimensional Fatigue Scale; PIFOS, Paediatric Injury Functional Outcome Scale; rHBI, Retrospective Health and Behaviour Inventory (rHBI). 
analysis with the household annual income and educational level variables.

\section{Balance error scoring system}

Balance will be evaluated by the RA using the Balance error scoring system (BESS), ${ }^{78}$ a widely used instrument in the field of sports concussion ${ }^{79}$ for objective assessment of postural stability. Two stances will be used: narrow double leg stance and tandem stance (hard surface only). Each stance is held, with hands on hips and eyes closed, for 20 s. Error points are given for specific behaviours, including opening eyes, lifting hands off hips or stepping, stumbling or falling. The BESS has shown satisfactory reliability in children and adolescents. ${ }^{80}$

\section{Physical activity questionnaire}

Preconcussion information on physical activity and sedentary habits will be assessed with selected questions from the Physical Activity and Children's Physical Activity surveys from the Canadian Health Measures Surveys (CHMS). The CHMS is a validated, reliable, standardised and comprehensive direct health measurement survey. ${ }^{81} 82$ These selected questions will provide a baseline measure of physical activity and sedentary behaviour habits, as these are possible confounding variables. Thus, the data collected with this assessment will be used to assess whether randomisation adequately accounts for potential differences in preconcussion activity levels. If physical activity habits are not adequately represented in the sample, it will be controlled for in our proposed analyses. In addition, by collecting this information, we will have the potential of comparing the results to a representative Canadian paediatric population from the CHMS.

\section{Sample size}

The sample size calculations for this trial were informed from unpublished data of the HBI assessment. Based on the parental HBI report, the head injury group had an HBI retrospective mean of 14.90; a 1-month HBI mean of $15.85(\mathrm{SD}=9.83)$ and a 12-month HBI mean of 13.22 (representing a return to normal). In the orthopaedic group, the retrospective parent-report mean was 13.38 , the HBI parental-report mean at 1 month was 13.29 and the HBI parental-report mean at 12 months was 11.64 . Considering these results, the experimental group is expected to demonstrate a $20 \%$ decrease in total symptom score at 2 weeks postinjury (ie, HBI $M=12.68$ ). Fixing the probability of type-I error at 5\%, assuming an SD of 9.83, a sample size of 152 per group would give $80 \%$ power to detect a $20 \%$ absolute difference in HBI total scores between the study arms. Accounting for an anticipated $15 \%$ loss in the follow-ups, a sample size of 175 per group is required (total number of 350).

\section{PROCEDURE}

\section{Data collection methods}

When a child with head injury presents to ED triage, the nurse will triage the child as per the existing protocol at each site. If the child has suffered symptoms possibly consistent with concussion, a member of the ED clinical team will inform the family that there is a study on children who have head injuries and ask if they are interested in learning more about the study. If so, the charts of potentially eligible patients will be flagged and the research team will be notified.

In families that agree to learn more about the study, the RA will introduce the study and complete a brief screening questionnaire. Patients who are not eligible based on initial screening will be thanked for their time. Eligible and willing parents, along with children and adolescents capable of consenting on their own behalf, will be asked for written informed consent; children aged 10 years or older unable to consent on their own behalf will be asked for assent. Subsequently, RAs will begin assessments and interventions. RAs will complete the screening and assessment questionnaires on a tablet for all potentially eligible patients who present to the ED during the study enrolment hours.

\section{ED visit and randomisation \\ Description}

Once consent is obtained, RAs will enrol patients and assign patient ID numbers by clinical sites. Subsequently, the RA will collect contact information and the demographic, injury and symptom information. Afterwards, participants will be tested with the BESS, rHBI, HBI and five selected questions from the physical activity and sedentary behaviours questionnaire.

\section{Physician management and prognostications}

The treating physician will assess the child as per the normal operating procedures of the ED. Once a patient has given consent to participate in the study, the RA will liaise with the treating physician to notify them that the patient is enrolled in the study. The child will remain in the normal treatment queue, and participation in this study is not anticipated to lengthen their ED visit. The physician will complete a brief electronic survey on the same tablet computer to provide data about presenting symptoms and the physical examinations (eg, mental status change, neurological deficits and ED GCS).

\section{Randomisation, blinding and allocation}

RAs will log into the Research Electronic Data Capture (REDCap) to randomise the patient to the experimental or control group, via a randomisation table. The randomised sequence was created using R V.3.1.1 software and was stratified by sites and sex, with a 1:1 allocation using random blocks. Only the RA recruiting and evaluating the participant will be unblinded; other researchers will be blinded to the allocated intervention protocol. The intervention plan to which participants are randomised will be a hidden field within the database; unblinding will be permissible if serious adverse events occur.

Subsequently, RAs will provide patients with physical rest recommendations (either experimental or control), cognitive rest recommendations as per the Zurich ACE return-to-learn guidelines and verbal and written education materials for accelerometer use. A flow chart of the time sequence of events in the ED can be reviewed in figure 2 .

\section{Follow-up procedures}

\section{Telephone/survey communication}

During the ED visit, caregivers will be asked their preferred method of communication, email or telephone. Following discharge, enrolled patients will either be entered into an automated follow-up web survey using REDCap ${ }^{83}$ or be contacted by telephone. Follow-up will be daily for 14 days (including a longer survey at the first and second week postinjury) and at the fourth-week postinjury for further data collection of primary and secondary outcome measures. A standardised script and survey/data collection form will be employed. Follow-up surveys include a daily logbook survey, the HBI, PCSI, PedsQL and PIFOS. 


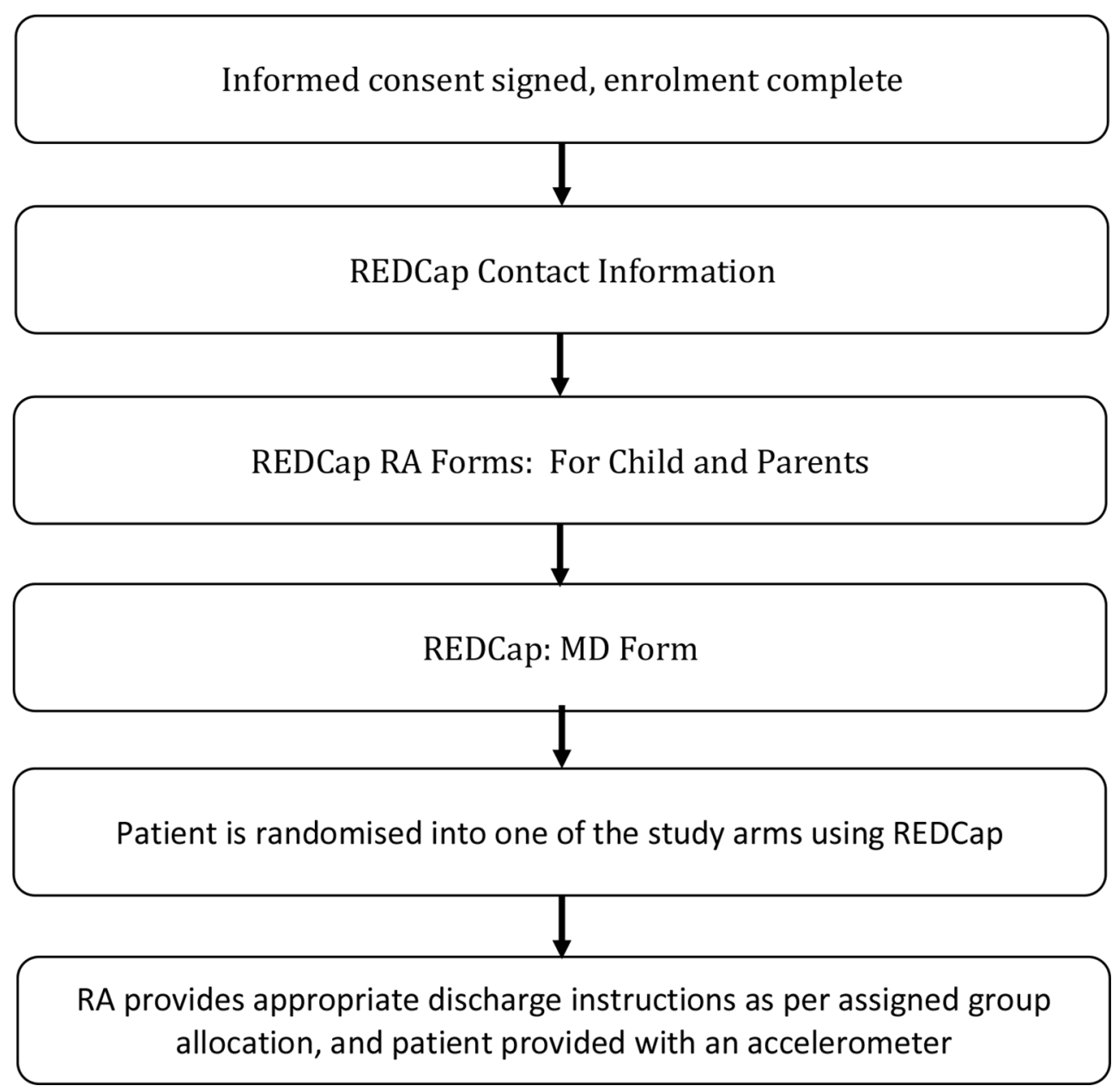

Figure 2 Flow chart of the sequence of event during the ED visit. ED, emergency department; RA, research assistant; REDCap, Research Electronic Data Capture.

Daily movement measurement

During the ED visit, all participants will be instructed on how to use the Actical accelerometer. All participants will be required to wear the accelerometer, for 14 consecutive days, 24 hours a day (removing only for water-related activities). To prevent reactivity to the measure, the device will be automatically activated at midnight. The accelerometer will be worn at the waist on an elasticised belt and positioned in line with the right mid-axillary line. Data will be collected at a sampling mode of $1 \mathrm{~min}$. Data will be automatically stored in memory on the accelerometer. On the 15 th day, participant will be instructed to return the Actical and belt via a courier service. Data will be downloaded directly into the REDCap database.

\section{Data management}

The Clinical Research Unit Data Coordinating Centre will be used as a central location for data processing and management, and data will be private and secured to industry standards for both clinical and patient sensitive data in Canada. Study data will be collected and managed using REDCap ${ }^{83}$ electronic data capture tools hosted and supported by the CHEO Research Institute. REDCap is a secure, web-based application designed to support data capture for research studies.

Only members of the research team will be granted access to the database. Users will be assigned to 'Data Access Groups' that will restrict their rights to viewing and entering data for their site alone. For monitoring purposes, the study coordinator will be able to view data from all sites. Within the Data Access Group, user privileges will be designated by the study coordinator to ensure research team members have only the minimum required rights to perform their duties. All identifying information that is collected will be flagged in the database and removed from data export unless the identifying information is required for statistical analysis (eg, date of birth).

\section{STATISTICAL ANALYSIS}

\section{Proposed analyses}

All main efficacy analyses will be based on the intent-to-treat principle. We will perform per-protocol analyses for additional insight. All reports and publications will distinguish these analyses. Multiple imputation techniques will be employed to replace data missing at random.

\section{Analysis of primary outcome}

The primary outcome investigated is whether early resumption of physical activity can reduce ratings of the frequency of PPCS compared with rest until resolution of symptoms at 2 weeks postinjury. The primary outcome will be evaluated with a linear multivariable model, where the total score on the HBI will be the dependent variable and treatment the primary independent variable of interest. The model will be adjusted for site and prognostically important covariates derived from Zemek et $a l^{8}$ : sex, age, prior concussion and symptom duration, migraine history, balance 
error scoring, answering questions slowly, headache, sensitivity to noise and fatigue. Assessment of collinearity using the variance inflation factor (VIF) will be performed, and if VIF greater than five is detected, variables will either be removed from the model or combined. Finally, main effect results with two-sided p values less than 0.05 will be considered to be statistically significant.

\section{Planned subgroups analyses}

Four planned linear models will be generated for the subgroup analyses for the primary outcome. (1) treatment $\times$ sex predicting PPCS-while concussion incidence is greater in males (approximately 60\%), evidence demonstrates a greater PPCS risk in females. ${ }^{84}$ Since concussion affects both sexes differently, the interaction between sex and treatment will be analysed. (2) Treatment $\times$ age (10-12 years, 13-17 years) predicting PPCS-evidence from Zemek et $a l^{8}$ demonstrated a greater PPCS risk in adolescents compared with younger children. To understand the optimal timing of reintroducing physical activities by age, the interaction effect between age and treatment will be investigated. (3) Treatment $\times$ severity of symptoms predicting PPCS—examining the relation between treatment and symptom severity predicting PPCS will be studied. (4) PPCS risk-a subgroup analysis stratified to PPCS risk using the PPCS prediction rule derived from the 5P study ${ }^{8}$ to stratify risk will be performed. A small difference in physical activity between treatment groups is expected for children with a quick recovery; the largest treatment difference is expected in children with protracted symptom recovery (elevated PPCS risk). Tests related to all subgroup analyses will be conducted with adjusted significance levels in order to keep the overall type I error rate less than 0.05 .

\section{Analysis of secondary outcomes}

Differences between the groups in health-related quality of life (PedsQL and PedsQL fatigue scale), functional outcome (PIFOS) and performance, sports/recreational participation and performance (data collection form collected in the ED), overall symptom burden (rHBI with HBI score at 1, 2 and 4 weeks and PCSI score at 2, 4 weeks), accelerometer data and adverse event rates will be analysed using parametric and non-parametric tests. We will also report observed differences in the secondary outcomes and corresponding 95\% using the appropriate summary statistic. We will use Holm's correction, as necessary, to correct for multiple testing.

\section{Analysis of secondary outcomes: ICES linkage}

As part of this novel study, with data linkage through use of the patient's OHIP number (with consent to use), the long-term healthcare use of concussion of both groups as well as the risks for concussion-related comorbidities (eg, mental health) group differences will be analysed. Future opportunities exist for ICES linkage to educational databases; a metadata analysis will permit examination of effect on school performance. Finally, we will be able to establish the economic effect related to paediatric concussion through ICES analysis.

\section{DATA SAFETY MONITORING}

\section{Data safety monitoring board}

The independent data safety monitoring board (DSMB) will consist of an independent paediatric ED physician, two paediatric concussion experts and a statistician not involved in the project. The DSMB will be immediately advised of severe adverse events, and they will meet in the event of a serious adverse event. Given the low risk intervention and no intention to stop the trial for benefit/futility, only biannual meeting will be required.

\section{Proposed frequency of analyses}

In keeping with StaRChild Health guidelines, ${ }^{85}$ the DSMB will, in collaboration with the trial steering committee, establish safety outcomes (including reinjury and PPCS rate and frequency rate at 4 weeks) and stopping rules prior to trial initiation. The DSMB will meet biannually to review enrolment, study procedures, form completion, data quality, loss to follow-up and interim safety results. Based on trends and adverse events, the DSMB may decide to meet sooner than planned using boundaries adjusted accordingly and may request unblinding if deemed necessary.

\section{Potential harms}

Safety outcomes include (1) repeated concussion or other exercise-related injuries within 4 weeks postinjury, (2) PPCS frequency rate at 4 weeks postinjury, (3) severe worsening of symptoms and (4) any symptoms requiring an unscheduled visit to the ED or primary care provider.

Although participants may experience an exacerbation of symptoms with increased activity, there are also risks associated with prolonged inactivity, including an increase in postconcussion symptoms. Participants in the experimental group will be instructed to progress with activity and exertion only if symptoms remain tolerable. Participants in the control group will be instructed to progress with activity and exertion only once asymptomatic. The activities undertaken in both groups are non-contact and as such pose little risk of head injury.

If concussion symptoms worsen during the treatment to the degree that the participant requires an unscheduled visit to the ED or primary care provider, then symptoms will be considered as an adverse event. Symptoms could develop into an adverse event if they become unbearable and the patient needs immediate medical attention. Patients will report adverse events through REDCap or the daily telephone survey. Daily reports will be monitored by RAs. In case of adverse events, site investigators will be notified in order to monitor the event and advise their respective REB, CHEO REB and DSMB.

\section{ETHICS AND DISSEMINATION \\ Ethical considerations}

The clinical trial study as received written approval from all sites REB. The study will be carried out according to the principles outlined in the Declaration of Helsinki, ${ }^{86}$ Good Clinical Practices, within the laws and regulation of the Tri-Council Policy Statement and the institutional policies of the CHEO REB.

Informed consent will be sought from all parents and participants aged 16 years and older. In addition, informed assent will be sought from participants under the age of 16 years, or from those deemed by their physician and the RA to be cognitively unable to provide informed consent.

\section{Confidentiality considerations}

Research personnel will take all appropriate and customary steps to ensure that data remain secure and that patient privacy and confidentiality are maintained.

\section{Dissemination}

Results will be disseminated at international conferences and in manuscripts to peer-reviewed journals. A manuscript communicating the results of the primary objective will be published in a peer-reviewed journal. Separate manuscripts will be written on each of the subgroup analyses and on the secondary 
objectives of the protocol, and these will also be submitted for publication in peer-reviewed journals. Finally, results will be integrated into concussion guidelines and shared with relevant key stakeholders (eg, public health agencies, education and sport ministries, sporting bodies, medical professional associations and foundations/associations encompassing sport and brain injury).

\section{DISCUSSION}

The proposed study is an innovative, large-scale, multicentre RCT, comparing two physical activity protocols: early resumption of non-contact aerobic physical exercise at 72 hours postinjury versus graduated return-to-play initiated only after full symptom resolution (rest until symptom resolution). The main objective of this study is to determine whether the early resumption of non-contact physical activity will yield superior recovery with a reduced degree of PPCS following a concussion. Secondary aims are to evaluate the effect of early reintroduction of physical activity on HRQoL, functional outcome, sleep and long-term use of healthcare services. Participants in the experimental group are expected to have a reduced risk of PPCS; they will also demonstrate better quality of life, functional outcomes and sleep compared with the control group. Finally, prospectively, participants in the experimental group are predicted to show reduced use of healthcare services compared with the control group.

International concussion experts participating in the Predicting and Preventing Postconcussive Problems in Pediatrics $(5 \mathrm{P})^{85187}$ study determined that 72 hours postinjury was an acceptable time to initiate physical activities in the experimental group, in terms of promoting recovery but doing so safely. In patients with a stroke (a severe TBI), fewer days from onset of stroke symptoms to the initiation of exercise programs have been consistently associated with improved functional outcomes and shorter rehabilitation, particularly in those with more severe symptoms ${ }^{88} 89$ The 72 -hour window allows time to begin recovery from metabolic and autoregulatory changes in the acute postinjury phase and was deemed safe to resume light non-jarring activities.

While we anticipate good adherence to the assigned protocol, the possibility of decreased compliance exists in both arms. In the experimental group, children and/or families may experience problems with resuming or progressing physical activities due to higher than anticipated symptoms. Alternatively, children in the control group may not fully refrain from physical activities until completely symptom-free, as currently recommended in return-to-play protocols. Both groups may not fully comply with the provided cognitive return-to-learn advice, although this should occur equally among both groups given the randomised design. Measures have been put into place to minimise compliance problems by (1) providing comprehensive educational materials on physical and cognitive rest at the time of study enrolment at the ED and (2) regular follow-up. Finally, the act of wearing an accelerometer may result in better adherence to the assigned protocol and will enable us to perform objective per-protocol analyses in addition to intention-to-treat analyses.

It is anticipated that the results from this study will provide healthcare professionals with evidence on when and how best to reintroduce physical activities in a patient's treatment plan. The knowledge gained from this randomised clinical trial will help guide paediatric concussion management by healthcare providers, families and educators/coaches and may positively affect patient-care and healthcare costs.
Acknowledgements We would like to acknowledge the PERC PedCARE team members for their contribution to this protocol.

Contributors RZ and NR conceived the study. AAL, RZ, NR, NB, CD, SR, KB, AD, GS, $\mathrm{KJF}, \mathrm{KB}, \mathrm{KOY}$ and MST contributed to the study design and contributed to the writing of the trial protocol. AAL and RZ prepared the first draft of the manuscript, $A A L, N R$, $C D, S R, K B, A D, G S, K J F, K B, K O Y$ and MST contributed to editing the manuscript and read and approved the final manuscript.

Funding This work was supported by Ontario SPOR Support Unit - IMPACT-Child Health Award, the Ontario Neurotrauma Foundation, and the CHEO Foundation.

Disclaimer The authors have no conflict of interest to disclose.

Competing interests RZ is supported by a clinical research chair in pediatric concussion by the University of Ottawa Brain and Mind Research Institute.

Ethics approval CHEORI REB, SickKids REB and London Children Hospital REB.

Provenance and peer review Commissioned; externally peer reviewed.

(C) Article author(s) (or their employer(s) unless otherwise stated in the text of the article) 2019. All rights reserved. No commercial use is permitted unless otherwise expressly granted.

\section{REFERENCES}

1 McCrory P, Meeuwisse W, Dvorak J, et al. Consensus statement on concussion in sport- the 5th International Conference on Concussion in Sport held in Berlin, October 2016. Br J Sports Med 2017 (Epub a head of print).

2 Langlois JA, Rutland-Brown W, Wald MM. The epidemiology and impact of traumatic brain injury: a brief overview. J Head Trauma Rehabil 2006;21:375-8.

3 National Center for Injury Prevention. Report to Congress on Mild traumatic Brain Injury in the United States: Steps to Prevent a Serious Public Health Problem. Atlanta, GA, 2003.

4 Carey S, Morrish J. Concussions in Ontario. Ontario Injury Compass, Spring 2013. Toronto, ON: Parachute, 2013.

5 Carroll L, Rosner D. The Concussion Crisis: Anatomy of a Silent Epidemic. New York, NY: Simon \& Schuster, 2011.

6 McCrory P, Meeuwisse WH, Aubry M, et al. Consensus statement on concussion in sport: the 4th International Conference on Concussion in Sport held in Zurich, November 2012. Br J Sports Med 2013;47:250-8.

7 Te Ao B, Brown P, Tobias M, et al. Cost of traumatic brain injury in New Zealand: evidence from a population-based study. Neurology 2014;83:1645-52.

8 Zemek R, Barrowman N, Freedman SB, et al. Clinical risk score for persistent postconcussion symptoms among children with acute concussion in the ED. JAMA 2016:315:1014-25.

9 Ayr LK, Yeates KO, Taylor HG, et al. Dimensions of postconcussive symptoms in children with mild traumatic brain injuries. J Int Neuropsychol Soc 2009;15:19-30.

10 Barlow KM, Crawford S, Stevenson A, et al. Epidemiology of postconcussion syndrome in pediatric mild traumatic brain injury. Pediatrics 2010;126:e374-81.

11 Yeates KO, Kaizar E, Rusin J, et al. Reliable change in postconcussive symptoms and its functional consequences among children with mild traumatic brain injury. Arch Pediatr Adolesc Med 2012:166:615-22.

12 Yeates KO, Luria J, Bartkowski H, et al. Postconcussive symptoms in children with mild closed head injuries. J Head Trauma Rehabil 1999;14:337-50.

13 Burton LJ, Quinn B, Pratt-Cheney JL, et al. Headache etiology in a pediatric emergency department. Pediatr Emerg Care 1997:13:1-4.

14 Belanger HG, Vanderploeg RD. The neuropsychological impact of sports-related concussion: a meta-analysis. J Int Neuropsycho/ Soc 2005;11:345-57.

15 Babcock L, Byczkowski T, Wade SL, et al. Predicting postconcussion syndrome after mild traumatic brain injury in children and adolescents who present to the emergency department. JAMA Pediatr 2013;167:156-61.

16 Yeates KO, Taylor HG, Rusin J, et al. Longitudinal trajectories of postconcussive symptoms in children with mild traumatic brain injuries and their relationship to acute clinical status. Pediatrics 2009;123:735-43.

17 Zemek R, Clarkin C, Farion KJ, et al. Parental anxiety at initial acute presentation is not associated with prolonged symptoms following pediatric concussion. Acad Emerg Med 2013:20:1041-9.

18 Scorza KA, Raleigh MF, O'Connor FG. Current concepts in concussion: evaluation and management. Am Fam Physician 2012;85:123-32.

19 Korinthenberg R, Schreck J, Weser J, et al. Post-traumatic syndrome after minor head injury cannot be predicted by neurological investigations. Brain Dev 2004;26:113-7.

20 Babikian T, Satz P, Zaucha K, et al. The UCLA longitudinal study of neurocognitive outcomes following mild pediatric traumatic brain injury. J Int Neuropsycho/ Soc 2011:17:886-95.

21 Meehan WP, Bachur RG. Sport-related concussion. Pediatrics 2009;123:114-23.

22 Zemek R, Duval S, Dematteo C. Guidelines for Diagnosing and Managing Pediatric Concussion. Toronto, Canada: Ontario Neurotrauma Foundation, 2014.

23 Purcell LK; Canadian Paediatric Society, Healthy Active Living and Sports Medicine Committee. Evaluation and management of children and adolescents with sportsrelated concussion. Paediatr Child Health 2012;17:31-2 
24 Purcell L. What are the most appropriate return-to-play guidelines for concussed child athletes? Br J Sports Med 2009;43(Suppl 1):i51-5.

25 Lovell MR, Fazio V. Concussion management in the child and adolescent athlete. Curr Sports Med Rep 2008;7:12-15.

26 Harmon KG, Drezner JA, Gammons M, et al. American Medical Society for Sports Medicine position statement: concussion in sport. Br J Sports Med 2013;47:15-26.

27 Halstead ME, Walter KD; Council on Sports Medicine and Fitness. American Academy of Pediatrics. Clinical report-sport-related concussion in children and adolescents. Pediatrics 2010;126:597-615.

28 Davis GA, Purcell LK. The evaluation and management of acute concussion differs in young children. Br J Sports Med 2014;48:98-101.

29 Giza CC, Kutcher JS, Ashwal S, et al. Summary of evidence-based guideline update: evaluation and management of concussion in sports: report of the Guideline Development Subcommittee of the American Academy of Neurology. Neurology 2013:80:2250-7.

30 Echemendia RJ, Giza CC, Kutcher JS. Developing guidelines for return to play: consensus and evidence-based approaches. Brain Inj 2015;29:185-94.

31 DeMatteo C, McCauley D, Stazyk K, et al. Post-concussion return to play and return to school guidelines for children and youth: a scoping methodology. Disabil Rehabil 2015;37:1107-12

32 DeMatteo C, Stazyk K, Giglia L, et al. A balanced protocol for return to school for children and youth following concussive injury. Clin Pediatr 2015;54:783-92.

33 Schneider KJ, Leddy JJ, Guskiewicz KM, et al. Rest and treatment/rehabilitation following sport-related concussion: a systematic review. Br J Sports Med 2017;0:1-7.

34 Davis GA, Anderson $\mathrm{V}$, Babl FE, et al. What is the difference in concussion management in children as compared with adults? A systematic review. Br J Sports Med 2017;51:949-57.

35 Institute of Medicine (IOM) and National Research Council (NRC). Sports-Related Concussions in Youth: Improving the Science, Changing the Culture. Washington, DC, 2013.

36 Zemek R, Eady K, Moreau K, et al. Knowledge of paediatric concussion among frontline primary care providers. Paediatr Child Health 2014;19:475-80.

37 Zemek R, Eady K, Moreau K, et al. Canadian pediatric emergency physician knowledge of concussion diagnosis and initial management. CJEM 2015;17:115-22

38 Dematteo CA, Hanna SE, Mahoney WJ, et al. "My child doesn't have a brain injury, he only has a concussion". Pediatrics 2010;125:327-34

39 Carson JD, Lawrence DW, Kraft SA, et al. Premature return to play and return to learn after a sport-related concussion: physician's chart review. Can Fam Physician 2014:60:e310-e312-5.

40 Stoller J, Carson JD, Garel A, et al. Do family physicians, emergency department physicians, and pediatricians give consistent sport-related concussion management advice? Can Fam Physician 2014;60:548-50.

41 Kinnaman KA, Mannix RC, Comstock RD, et al. Management of pediatric patients with concussion by emergency medicine physicians. Pediatr Emerg Care 2014;30:458-61.

42 Zonfrillo MR, Master CL, Grady MF, et al. Pediatric providers' self-reported knowledge, practices, and attitudes about concussion. Pediatrics 2012:130:1120-5.

43 Brown NJ, Mannix RC, O'Brien MJ, et al. Effect of cognitive activity level on duration of post-concussion symptoms. Pediatrics 2014;133:e299-e304.

44 Thomas DG, Apps JN, Hoffmann RG, et al. Benefits of strict rest after acute concussion: a randomized controlled trial. Pediatrics 2015;135:213-23.

45 Shen X, Li A, Zhang Y, et al. The effect of different intensities of treadmill exercise on cognitive function deficit following a severe controlled cortical impact in rats. Int J Mol Sci 2013;14:21598-612.

46 Willer B, Leddy JJ. Management of concussion and post-concussion syndrome. Curr Treat Options Neurol 2006:8:415-26.

47 Griesbach GS, Hovda DA, Gomez-Pinilla F. Exercise-induced improvement in cognitive performance after traumatic brain injury in rats is dependent on BDNF activation. Brain Res 2009;1288:105-15

48 Leddy JJ, Kozlowski K, Donnelly JP, et al. A preliminary study of subsymptom threshold exercise training for refractory post-concussion syndrome. Clin I Sport Med 2010;20:21-7.

49 Gagnon I, Galli C, Friedman D, et al. Active rehabilitation for children who are slow to recover following sport-related concussion. Brain Inj 2009;23:956-64.

50 Gagnon I, Grilli L, Friedman D, et al. A pilot study of active rehabilitation for adolescents who are slow to recover from sport-related concussion. Scand J Med Sci Sports 2016;26:299-306.

51 Grool AM, Aglipay M, Momoli F, et al. Association between early participation in physical activity following acute concussion and persistent postconcussive symptoms in children and adolescents. JAMA 2016;316:2504.

52 Schneider KJ, Iverson GL, Emery CA, et al. The effects of rest and treatment following sport-related concussion: a systematic review of the literature. Br J Sports Med 2013:47:304-7.

53 Osmond MH, Klassen TP, Wells GA, et al. CATCH: a clinical decision rule for the use of computed tomography in children with minor head injury. CMAJ 2010;182:341-8

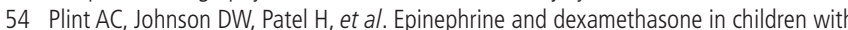
bronchiolitis. N Engl J Med 2009;360:2079-89.

55 Gioia GA. Multimodal evaluation and management of children with concussion: using our heads and available evidence. Brain Inj 2015;29:195-206.
56 Roemmich JN, Barkley JE, Epstein LH, et al. Validity of PCERT and OMNI walk/run ratings of perceived exertion. Med Sci Sports Exerc 2006:38:1014-9.

57 Iverson GL, Gioia GA. Returning to school following sport-related concussion. Phys Med Rehabil Clin N Am 2016:27:429-36.

58 McCauley SR, Wilde EA, Anderson VA, et al. Recommendations for the use of common outcome measures in pediatric traumatic brain injury research. J Neurotrauma 2012;29:678-705.

59 World Health Organization. The ICD-10 Classification of Mental and Behavioural Disorders: Clinical Descriptions and Diagnostic Guidelines. Geneva: World Health Organization, 1992.

60 Sady MD, Vaughan CG, Gioia GA. Psychometric characteristics of the postconcussion symptom inventory in children and adolescents. Arch Clin Neuropsychol 2014;29:348-63.

61 Gioia GA, Isquith PK, Schneider JC, et al. New approaches to assessment and monitoring of concussion in children. Top Lang Disord 2009:29:266-81.

62 Gioia GA, Schneider JC, Vaughan CG, et al. Which symptom assessments and approaches are uniquely appropriate for paediatric concussion? Br J Sports Med 2009;43(Suppl 1):i13-i22

63 Gioia GA, Isquith PK, Schneider JC, et al. New approaches to assessment and monitoring of concussion in children. Top Lang Disord 2009;29:266-81.

64 Desai AD, Zhou C, Stanford S, et al. Validity and responsiveness of the pediatric quality of life inventory (PedsQL) 4.0 generic core scales in the pediatric inpatient setting JAMA Pediatr 2014;168:1114-8.

65 Varni JW, Burwinkle TM, Seid M, et al. The PedsQL 4.0 as a pediatric population health measure: feasibility, reliability, and validity. Ambul Pediatr 2003;3:329-41

66 Adelson PD, Pineda J, Bell MJ, et al. Common data elements for pediatric traumatic brain injury: recommendations from the working group on demographics and clinical assessment. J Neurotrauma 2012;29:639-53.

67 Varni JW, Beaujean AA, Limbers CA. Factorial invariance of pediatric patient selfreported fatigue across age and gender: a multigroup confirmatory factor analysis approach utilizing the PedsQLTM multidimensional Fatigue Scale. Qual Life Res 2013;22:2581-94.

68 Varni JW, Burwinkle TM, Szer IS. The PedsQL Multidimensional Fatigue Scale in pediatric rheumatology: reliability and validity. J Rheumato/ 2004;31:2494-500.

69 Rivara FP, Koepsell TD, Wang J, et al. Disability 3, 12, and 24 months after traumatic brain injury among children and adolescents. Pediatrics 2011;128:e1129-38.

70 Ewing-Cobbs L, Prasad MR, Kramer L, et al. Late intellectual and academic outcomes following traumatic brain injury sustained during early childhood. J Neurosurg 2006:105:287-96

71 Ewing-Cobbs L, Fletcher JM, Levin HS, et al. Academic achievement and academic placement following traumatic brain injury in children and adolescents: a two-year longitudinal study. J Clin Exp Neuropsychol 1998;20:769-81.

72 Ewing-Cobbs L, Bloom DR, Prasad MR, et al. Assessing recovery and disability after physical trauma: the Pediatric Injury functional Outcome Scale. J Pediatr Psychol 2014;39:653-65

73 Jaffe KM, Polissar NL, Fay GC, et al. Recovery trends over three years following pediatric traumatic brain injury. Arch Phys Med Rehabil 1995;76:17-26.

74 Puyau MR, Adolph AL, Vohra FA, et al. Prediction of activity energy expenditure using accelerometers in children. Med Sci Sports Exerc 2004;36:1625-31.

75 Evenson KR, Catellier DJ, Gill K, et al. Calibration of two objective measures of physical activity for children. J Sports Sci 2008;26:1557-65.

76 Tudor-Locke C, Barreira TV, Schuna JM, et al. Improving wear time compliance with a 24-hour waist-worn accelerometer protocol in the International Study of Childhood Obesity, Lifestyle and the environment (ISCOLE). Int J Behav Nutr Phys Act 2015:12:11

77 Tudor-Locke C, Camhi SM, Troiano RP. A catalog of rules, variables, and definitions applied to accelerometer data in the National Health and Nutrition Examination Survey, 2003-2006. Prev Chronic Dis 2012;9:E113.

78 Guskiewicz KM. Postural stability assessment following concussion: one piece of the puzzle. Clin J Sport Med 2001;11:182-9.

79 Guskiewicz KM, Ross SE, Marshall SW. Postural stability and neuropsychological deficits after concussion in collegiate athletes. J Ath/ Train 2001;36:263-73.

80 Valovich McLeod TC, Barr WB, McCrea M, et al. Psychometric and measurement properties of concussion assessment tools in youth sports. J Athl Train 2006;41:399-408.

81 Tremblay M, Wolfson M, Connor Gorber S. Canadian health measures survey: rationale, background and overview. Health Rep 2007;18(Suppl):7-20.

82 Tremblay MS, Shields M, Laviolette M, et al. Fitness of Canadian children and youth: results from the 2007-2009 Canadian Health Measures Survey. Health Rep 2010;21:7-20

83 Harris PA, Taylor R, Thielke R, et al. Research electronic data capture (REDCap) - a metadata-driven methodology and workflow process for providing translational research informatics support. J Biomed Inform 2009;42:377-81.

84 Zuckerman SL, Apple RP, Odom MJ, et al. Effect of sex on symptoms and return to baseline in sport-related concussion. J Neurosurg Pediatr 2014;13:72-81.

85 Sampson MR, Benjamin DK, Cohen-Wolkowiez M. Evidence-based guidelines for pediatric clinical trials: focus on StaR Child Health. Expert Rev Clin Pharmacol 2012;5:525-31. 
86 World Medical Association. World Medical Association declaration of Helsinki: ethical principles for medical research involving human subjects. JAMA 2013;310:2191-4.

87 Zemek R, Osmond MH, Barrowman N; Pediatric Emergency Research Canada (PERC) Concussion Team. Predicting and preventing postconcussive problems in paediatrics (5P) study: protocol for a prospective multicentre clinical prediction rule derivation study in children with concussion. BMJ Open 2013;3:e003550-10
88 Maulden SA, Gassaway J, Horn SD, et al. Timing of initiation of rehabilitation after stroke. Arch Phys Med Rehabil 2005;86:34-40.

89 Horn SD, DeJong G, Smout RJ, et al. Stroke rehabilitation patients, practice, and outcomes: is earlier and more aggressive therapy better? Arch Phys Med Rehabil 2005;86:101-14. 